\title{
Implementation of Generic Multi-Purpose Robotic Arm
}

\author{
${ }^{1}$ Abhishek Chavan, ${ }^{2}$ Abhishek Bhuskute, ${ }^{3}$ Anmol Jain, ${ }^{4}$ Neha Shinde, ${ }^{5}$ Mahendra Salunke \\ Dept. of Computer Engineering \\ Sinhgad Institute of Technology \& Science \\ Pune, India
}

\begin{abstract}
This decade of electronic and computer advancements have aided society significantly due to interdisciplinary approach. It implies growth in fields like 'Embedded Systems' and 'Artificial Intelligence'. This paper describes use of programmed intelligence of a robotic arm that assists imitating human arm, pick and drop operation, tracing trajectories and simulating learning algorithm. Unlike other domain-specific robotic arms, this paper proposes a multi-purpose mobile assembly mounted on wheels. The assembly model is based on human arm as it has rotational ability at wrist, elbow and shoulder. Actuators at each joint carry the torque generated by payload and overall arm load. This paper describes prototype that executes 'Reinforcement Learning' and shows that such assembly can effectively run complex algorithms. The functions specified at a higher abstract level make it a generic system.
\end{abstract}

\section{Keywords}

Robotic Arm, Imitation, Artificial intelligence, Arduino,Trajectory Tracing

\section{INTRODUCTION}

The manually operated robots have aided many industries as they reduce life casualties and financial degradation while performing risky and critical operations. But operating these machines needed manual synchronization of actuators and was considered tedious task. Therefore, the automated machines are popular nowadays in industries. But area of operation still remains narrow. These are domain-specific machines which need accurate technical feed. This paper presents a prototype that demonstrated an intuitive and user friendly system. A robotic arm that offers functions specified at higher level of abstraction and transparently asserts actuators based on programmed commands. $[1]$

This prototype can be extended to applications where imagination is the limit. With superior actuators, supporting assembly of processors and batteries, it can overcome human strength, speed, accuracy and precision.[4] The prototype uses Bluetooth interfacing of sensors with controller board. It suggests that the assembly may perceive the context from distance to perform action based on event. An assembly is mounted on wheels so as to enable the robotic arm to move around. With this feature, the mechanism becomes support to physically disabled people. There are distinct modes of operation of robot that make it behave in a manner consistent with user requirement. The mode can be set wirelessly with the help of an IR Remote. The panel of sensors is a wearable set that has to be equipped by human controller. The same set is used to drive robotic arm in various operational modes. For example, in imitation mode, a robotic arm will typically imitate the human arm movements and turning locomotion mode on will read wrist gyro sensors to move the entire assembly in desired manner.

\section{LITERATURE SURVEY}

\section{1. "The Multiple-Function Intelligent} Robotic Arms"

The main design goal of this robotic arm was to present the following functions: Chinese calligraphy, fancy dancing, weightlifting and color classification. Another design goal was to minimize cost and maximize performance.[5]

Hence it is designed in a way so as to perform many tasks as a human arm. The simplicity of its design gave it the way to perform motion as good as a human arm with improved values of strength and speed.[5]

\section{2. "Development of a robotic-arm} controller by using hand gesture recognition" This robotic arm controller is using image processing in the field of Human-Machine Interaction. There are two methods used to control the robotic arm, the main task of them is to get the hand gesture data without using tool that helps the system to extract data easier (ex. glove or wrist band). The subsequent operations are decided based on these changes.[6]

The first method is comparing of all pre-stored data in the database at the Template Matching Algorithm, the second method is Signature Signal, distance signal between edge of the hand and centre of hand. These necessarily use the gyro-sensors as the computation of change in angular values shall be obtained at time of operation.[6]

\section{3. "The development of six D.O.F. robot arm for intelligent robot"}

The aim of this paper is to develop a six D.O.F robot arm for an intelligent robot. As for the base or shoulder part, the harmonic responding drive, which has some advantages, was used to ensure the weight of the whole arm could be supported. This provides a degree of freedom along with supporting the assembly. The kinematic equations of the system have been studied and shown in this. The degrees in which the robotic unit make moves or acquires motion necessarily contribute to improvement of operation. Every added or updated motion represents ease of operation in certain operating context. [7] At the end, we investigate some experiments; the computer system based controller can command the robot arm to pick a box through a moving path successfully.[7]

\section{MATHEMATICAL MODEL}

\subsection{Rotational motion of one degree of freedom}

The motion specified in Fig. 1 shows the manipulator considering an axis of reference and the respective trajectory of rotation 
The rotational trajectory is guided by angle $\theta(d)$ and length of links. The generalized trajectory of manipulation can be given by

$$
R(d)=\left[\begin{array}{ccc}
\cos \theta(d) & -\sin \theta(d) & 0 \\
\sin \theta(d) & \cos \theta(d) & 0 \\
0 & 0 & 1
\end{array}\right]
$$

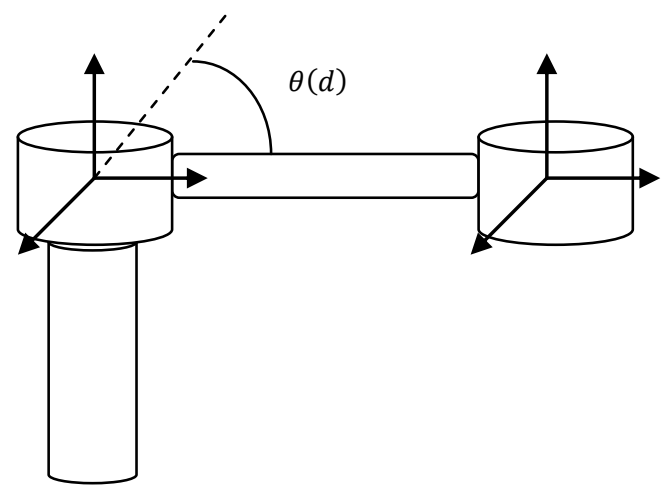

Figure 1: Rotational motion of one degree of freedom

\subsection{Positional Model}

As the prototype for robotic arm involves multiple pivots, we need to consider following model of rotation and length of links.

Considering 2-dimensional mathematical model so as to establish relational expression among $\theta 1, \theta 2$ and $\theta 3$ are the turning angles of three links respectively. $\mathrm{x}$ and $\mathrm{y}$ are the final positions of the multi link system. $\Phi$ is resultant of $\theta 1, \theta 2$ and $\theta 3$ angles. Following relation can be considered.

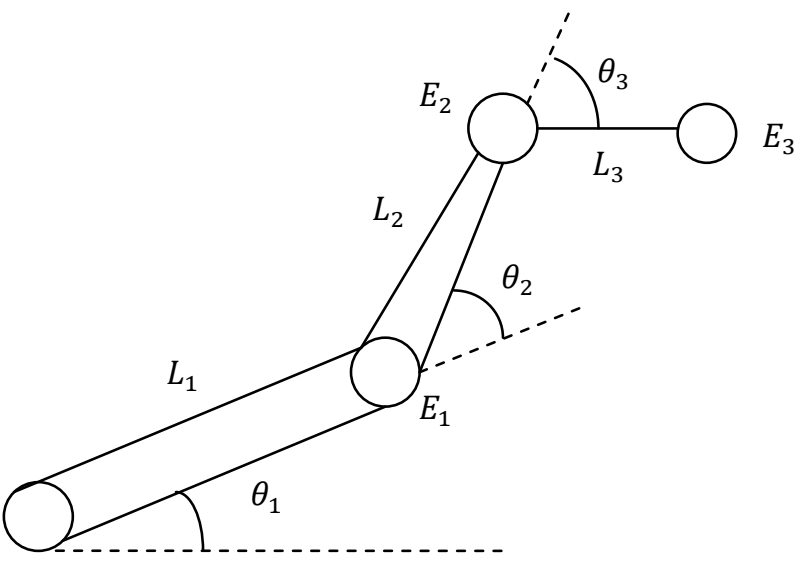

Figure 2: Positional Model

L1, L2 and L3 are the lengths of three links. In this case, the rotational matrices are as follows:

$$
\begin{aligned}
\text { Tix } & =\left[\begin{array}{ccc}
1 & 0 & 0 \\
0 & \cos \theta_{i} & -\sin \theta_{i} \\
0 & \sin \theta_{i} & \cos \theta_{i}
\end{array}\right] \\
\text { Tiy } & =\left[\begin{array}{ccc}
\cos \theta_{i} & 0 & \sin \theta_{i} \\
0 & 1 & 0 \\
-\sin \theta_{i} & 0 & \cos \theta_{i}
\end{array}\right]
\end{aligned}
$$

$L i=\left[\begin{array}{c}0 \\ 0 \\ L_{i}\end{array}\right]$

$E 1=T 1 x L 1$

$E 2=T 1 x L 1+T 1 X T 2 x L 2$

$E 3=T 1 x L 1+T 1 X T 2 x L 2+T 1 x T 2 x T 3 x L 3$

Where, E1, E2 and E3 are end positions of the links.

\section{METHODOLOGIES}

The prototype this paper describes uses simple methodologies that must be noted to understand its behavior. The main concerns of model are to keep the prototype user friendly, generic, multipurpose and compact. These methodologies are implemented to simulate human-like actions.

\subsection{Interpolation method for guiding motion}

There is a significant difference observed in human arm and that of guided robotic arm. A typical robotic arm gets to specified position by making changes in pivots one after the other. Even if motors are synchronized for a particular destination, it does so with jerky moves. To enable such a machine to draw shapes and simulate natural human-like moves, an approach of interpolation can be used.

Interpolation is a method of obtaining or marking a set of points in a trajectory from a given set of points. Now the trajectory obtained if it is guided by given set of points is given as,

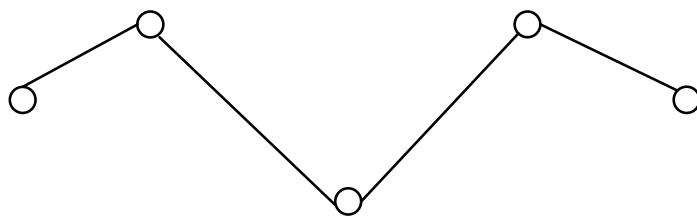

Figure 3: Motion without interpolation

However, a natural motion will involve a curve trajectory if motion has to be guided by set of points. It typically takes following form,

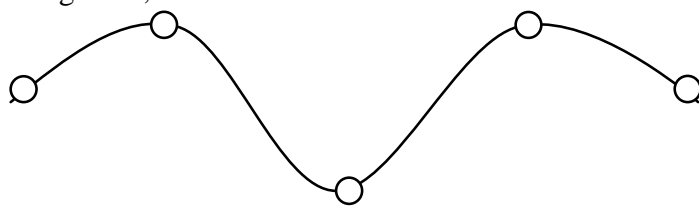

Figure 4: Motion using interpolation

Therefore it is essential to use a suitable interpolation function so as to enable machine to obtain smooth movements.

\subsection{Trajectory tracing in 3D Context}

In order to trace a path in 3D context, a machine must reach to desired point specified in co-ordinate system. A point can be specified in co-ordinate system as following Fig. 5.

Assume that the base of arm is at point $\mathrm{A}(0,0,0)$ and point $\mathrm{D}(3,3,4)$ is destination point where tip of arm is supposed to reach. The arm must be brought in the plane from where the tip of arm can reach to destination point $D$. Hence we rotate base by desired angle $\alpha$ (Consider top view) in Fig. 6.

$$
\alpha=\tan ^{-1}\left(\frac{B C}{A B}\right)=\tan ^{-1}\left(\frac{y}{x}\right)
$$




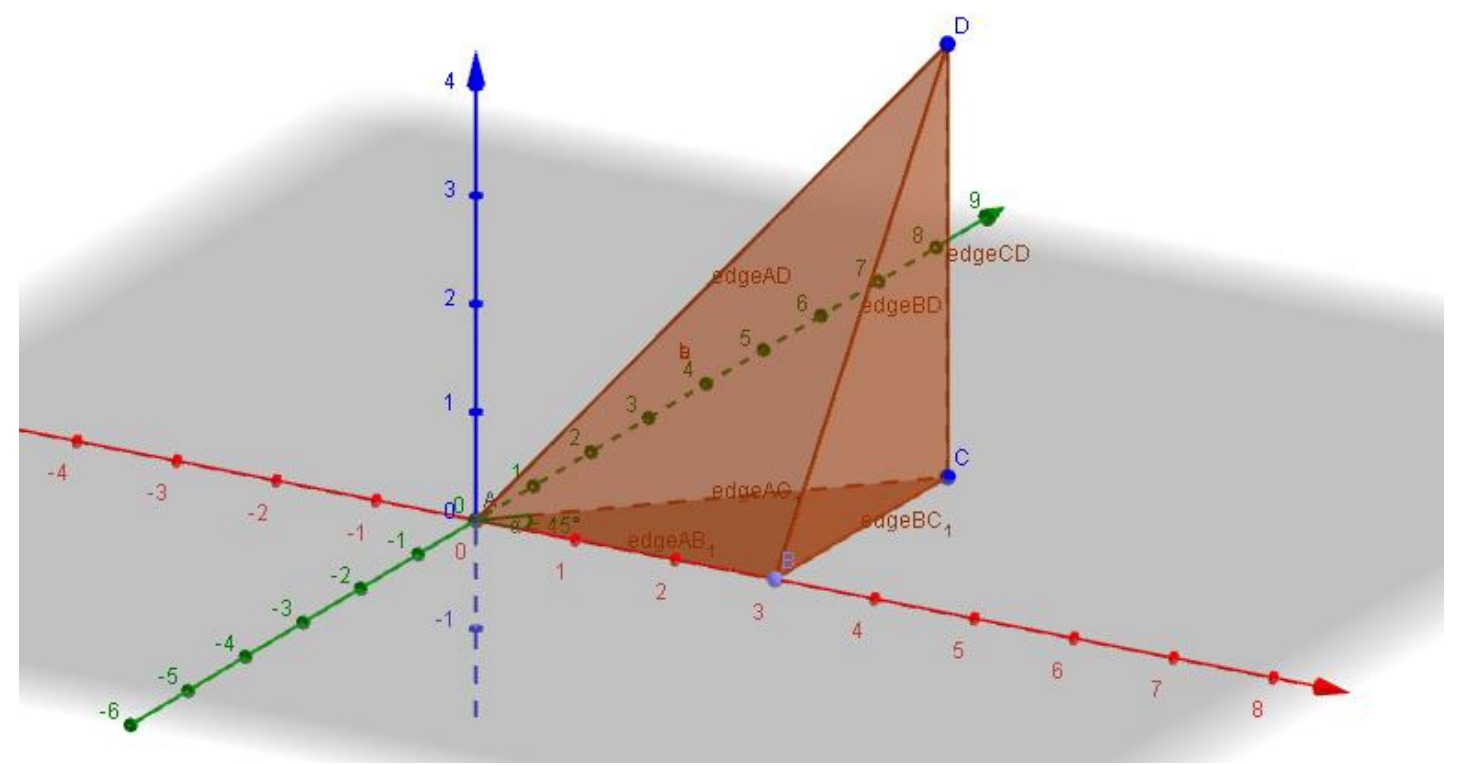

Figure 5: Isometric view of 3D context

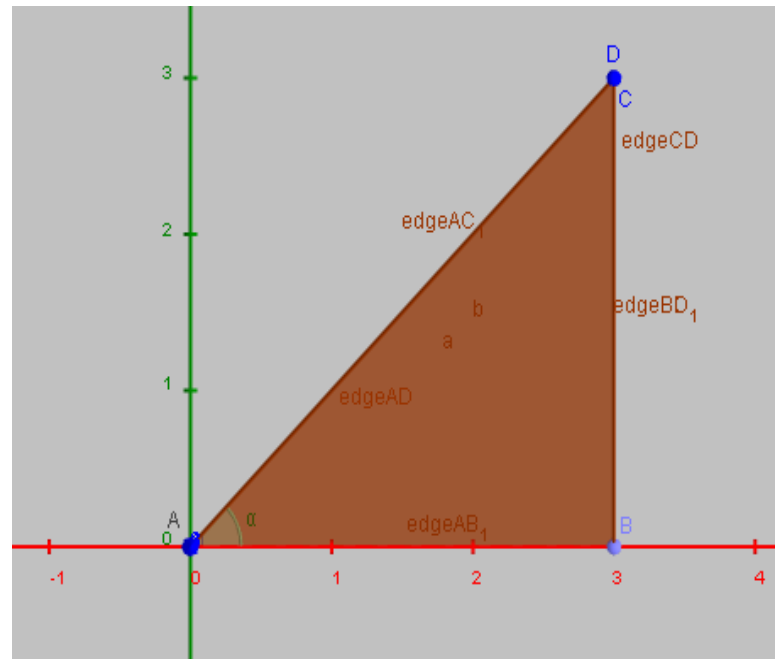

Figure 6: Top view of 3D context

Once $\alpha$ has been set, mapping can be confined only to a single plane. This needs considering ACD plane. Refer Fig. 7.

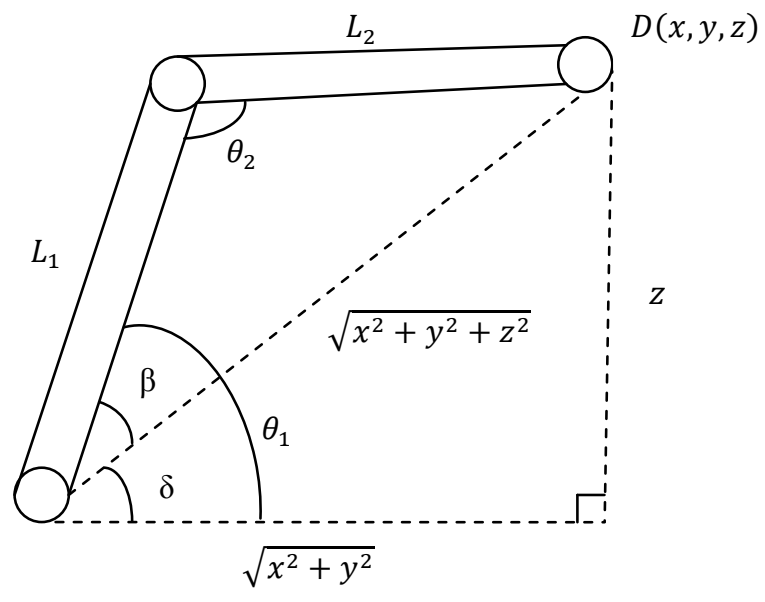

Figure 7: Side view of 3D context
In above figure, all segment lengths are known, therefore we get values for angle $\beta$ and $\theta_{2}$ by using cosine rule.

$$
\begin{gathered}
\delta=\tan ^{-1}\left(\frac{z}{\sqrt{x^{2}+y^{2}}}\right) \\
\theta_{1}=\beta+\delta
\end{gathered}
$$

Further, these values can be set by motors to guide tip of arm to destination point.

By programming required interpolation method, desired curves can also be traced. This scope can be extended to enable machine to draw as like human.

\subsection{Bluetooth communication}

The implementation can effectively communicate using Bluetooth system. There is a panel of sensors with controller that collect required data. This perception has to be delivered to the arduino located on the robotic arm assembly.

The Bluetooth unit at controller's end encapsulates the collected data in a packet as specified in Fig. 8 and sends it to arduino board. An on-board running program reads this packet and required action is taken based on received input. There are many choices for carrying out communication. It can be customized based on needs for area of application. The communication system remains independent from rest of operational system. One may use Wi-Fi to overcome constraint on distance or IR signaling for cost reduction. The Bluetooth enables remote machine to be a smart phone or Bluetooth equipped gadget.

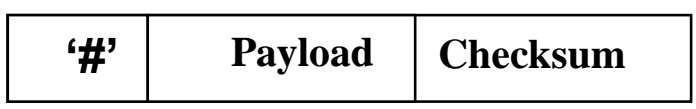

Figure 8: Packet format

Each packet is initialized with a '\#' byte to avoid reception of false data. Then the payload section contains all required data values that have been read from sensors. Finally, a checksum is included in each packet to verify its validity. 


\subsection{Arduino IDE}

Arduino IDE offers a well-structured wide range of functions those direct the arduino pins and ports transparently. Arduino IDE facilitates programming Arduino boards with simplicity and writing those on arduino board. There is a SERVO library of functions that can be used to access Servo motors. It reduces the programming overhead and improves running time of underlying program.

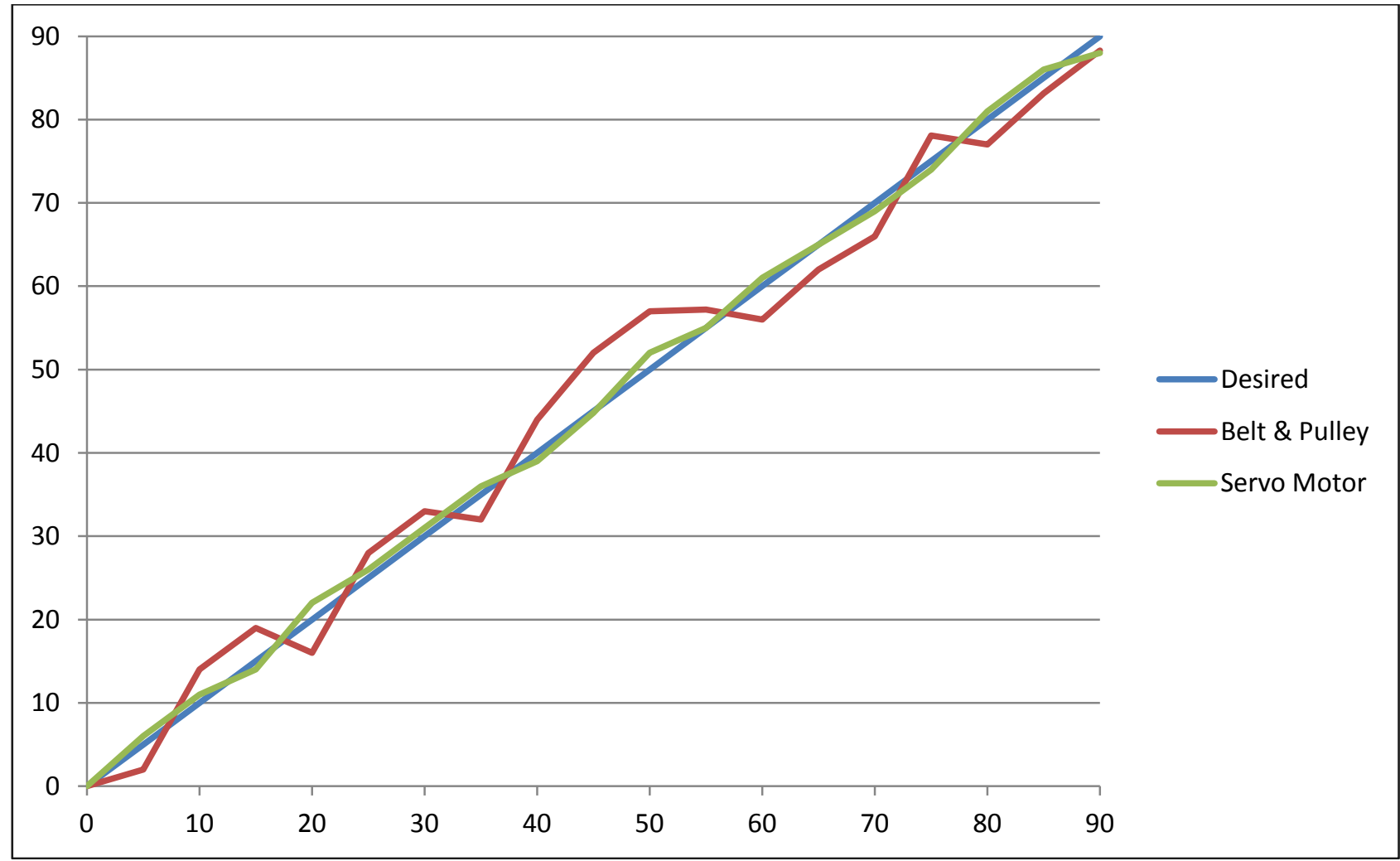

Figure 9: Chart comparison of implementation techniques

\section{MECHANICAL STRUCTURE}

\subsection{The Servo and DC motors}

This prototype has been driven by a set of 8 Servo motors and 4 DC motors. Previously the Belt and Pulley mechanism was installed to carry out entire arm motion. Later it was replaced by servo motors. Significant improvement was seen in accuracy with this replacement.

The purpose specific machines are relatively less complicated and more efficient. To provide a substantial degree of precision, the accuracy of angular moves is necessary. In proposed robotic arm prototype the rotatable base contributes to a major degree of accuracy.

The degree of accuracy increases if rotatable base is assisted by using Servo motors with arduino servo library of functions. The relative degree of accuracy using belt and pulley assembly was less. The following results illustrate the individual accuracy of both techniques. In plotting shown below, linearity represents precision of motors and values are to be compared to desired results so as to evaluate degree of accuracy. Refer Fig. 9.

In Fig. 9, the units of both axes are angles in Degree values.
Table 1: Comparison of implementation techniques

\begin{tabular}{|c|c|c|}
\hline Programmed & $\begin{array}{c}\text { Belt \& } \\
\text { Pulley }\end{array}$ & $\begin{array}{c}\text { Servo } \\
\text { Motor }\end{array}$ \\
\hline 0 & 0 & 0 \\
\hline 5 & 2 & 6 \\
\hline 10 & 14 & 11 \\
\hline 15 & 19 & 14 \\
\hline 20 & 16 & 22 \\
\hline 25 & 28 & 26 \\
\hline 30 & 33 & 31 \\
\hline 35 & 32 & 36 \\
\hline 40 & 44 & 39 \\
\hline 45 & 52 & 44.8 \\
\hline 50 & 57 & 52 \\
\hline 55 & 57.2 & 55 \\
\hline 60 & 56 & 61 \\
\hline 65 & 62 & 65 \\
\hline 70 & 66 & 69 \\
\hline 75 & 78.1 & 74 \\
\hline & & \\
\hline
\end{tabular}




\begin{tabular}{|c|c|c|}
80 & 77 & 81 \\
\hline 85 & 83.13 & 86 \\
\hline 90 & 88.3 & 88 \\
\hline
\end{tabular}

\subsection{Prototype body}

The body of prototype was designed in Corel Draw designing tool. The output file (.cdr) is treated as a prototype design. After several rounds of design optimization, an acrylic material was used to build robotic arm model. File with .cdr preserves the actual dimensions of the design. This file was used for laser cutting of the acrylic to maintain precise shape and size of the prototype.

\section{CONCLUSION}

This paper presents the generic robotic arm model which drags application area out of domain-specific operations. It suggests with mentioned prototype that these machines are well suited and reliable for several common tasks. If equipped with powerful motors, processors and batteries, these machines will definitely assist human in day-to-day life. Such a user friendly system can be useful to overcome strength, speed, precision and accuracy barriers. The prototype is capable of executing complex algorithms as it has successfully demonstrated reinforcement learning.

The implementation leads to construction of a library of abstract functions those enable programming these machine an interactive and easy task. The details regarding these machines are not essential while specifying actions to be done. This in turn builds a platform that certainly accelerates further research and development in this field.

Implementation of generic model can be programmed with interactive algorithms those enable machines to learn routines and actions quite effectively. These machines can behave in a manner that adapts its context and execute suitable behavior. This makes it a versatile and robust entity in itself.

\section{FUTURE SCOPE}

As the behavior of such robotic assemblies can be governed using mathematical models and algorithms applied to behave in a manner consistent with environment, these assemblies are suitable to perform adaptive actions.

These machines are suitably driven by arduino, beaglebone or a microcontroller assembly. Hence they can be equipped with learning algorithms such as reinforcement learning. This aspect has to be developed in every possible way to identify and extract useful features of these assemblies.

Further, the machine can be equipped efficiently with BrainComputer Interfacing (BCI) technology. There are open- sourced hardware and software resources available and a wide so as to have an incremental platform of development.

The machines can also be assisted using voice-recognition. These provide an edge of advantage for physically challenged people. Such collaborations make these assemblies a customizable asset. Therefore, the system is truly scalable and collaboration with new and upcoming technologies is possible.

\section{ACKNOWLEDGMENT}

We would like to thank Mr. Deepak Dalai for his contribution in offering guidance for this project. We also thank Dept. of Computer Engineering, SITS, Pune, India for valuable suggestions.

Prof. Mr. Nihar Ranjan worked as project coordinator and extended help to complete this project in timely manner. We would like to thank him for his advice and guidance.

This work is sponsored by CORORES, Pune, India.

Abhishek Chavan, Abhishek Bhuskute, Anmol Jain and Neha Shinde are currently pursuing degree in Computer Engineering at SITS, Pune, India.

Prof. Mr. M. B. Salunke is currently working at SITS, Pune, India.

\section{REFERENCES}

[1] Gregorio Juliana, Álvaro Rodríguez, Hugo Ramos, "Development of a multi-purpose, modular, low cost robotic arm for education and unmanned platforms".

[2] Arduino Foundation Website, arduino Uno and Nano schematics, http://arduino.cc/en/tutorial/foundations

[3] Arduino Software as Arduino IDE, http://arduino.cc/en/main/software

[4] Celik, I.B. ; Dept. of Electr. Electron. Eng., Dokuz Eylu Univ., Izmir, Turkey; Kuntalp, M., "Development of a robotic-arm controller by using hand gesture recognition".

[5] A"The Multiple-Function Intelligent Robotic Arms", Rong-Jyue Wang, Jun-Wei Zhang, Jia-Ming $\mathrm{Xu}$, and Hsin-Yu Liu.

[6] "Development of a robotic-arm controller by using hand gesture recognition", Celik, I.B. ; Dept. of Electr. Electron. Eng., Dokuz Eylul Univ.,Izmir, Turkey; Kuntalp, M.

[7] "The development of six D.O.F. robot arm for intelligent robot", Jie-Tong Zou ; Dept. of Aeronaut. Eng., Nat. Formosa Univ., Huwei Township, Taiwan; Des-Hun Tu. 
APPENDIX

1. Working circuit implementation

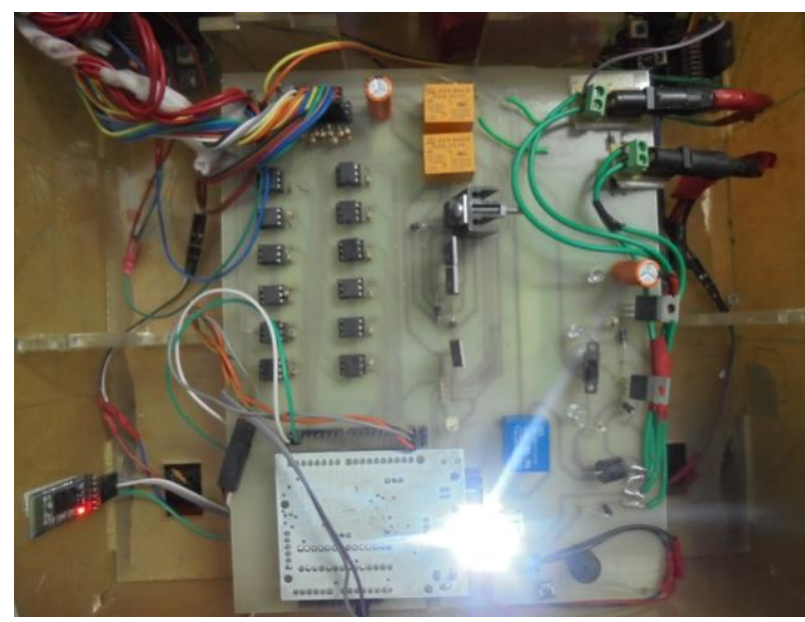

1. Human arm imitation

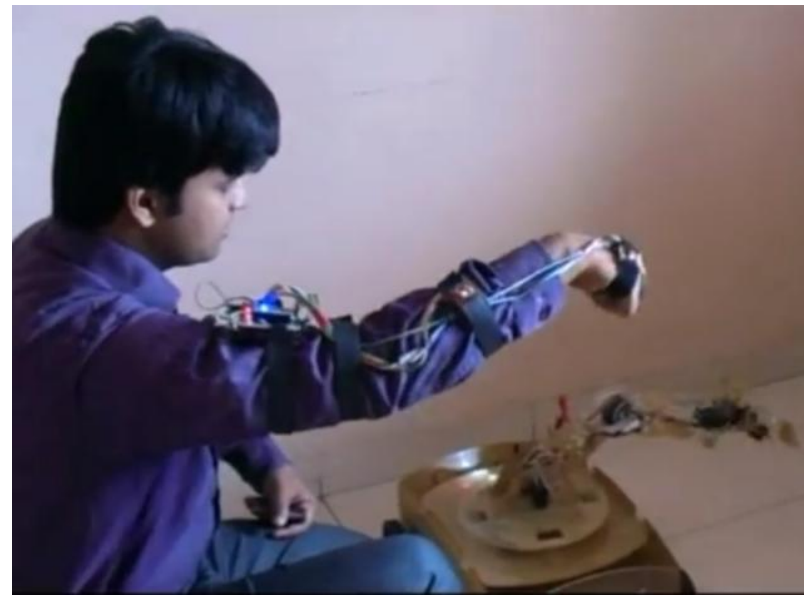

2. Built acrylic prototype

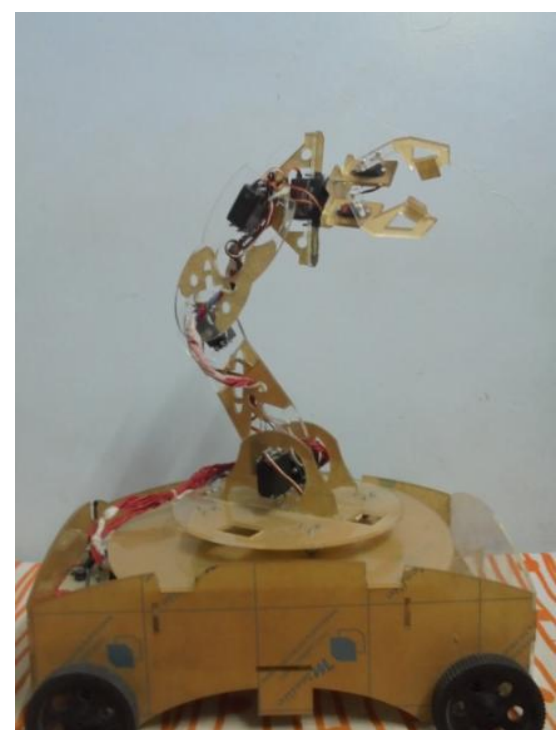

3. Locomotion assessment

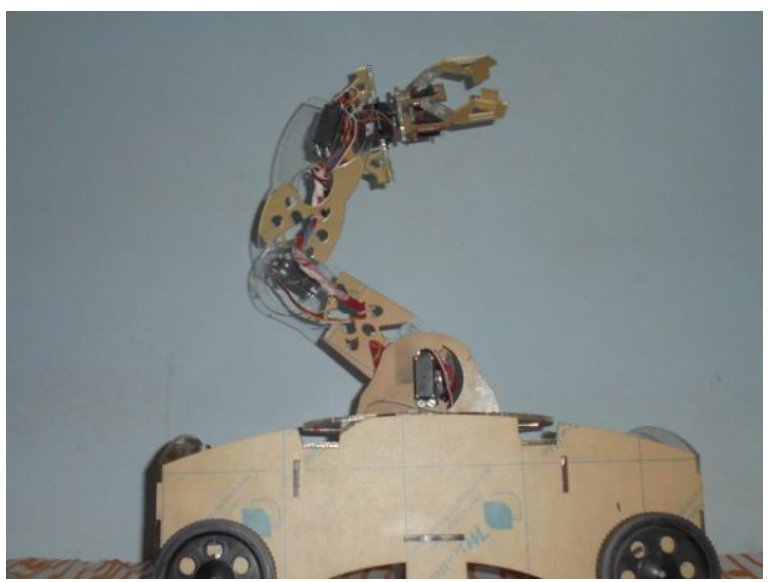

